\title{
Extremality conditions for generalized channels
}

\author{
Anna Jenčová* \\ Mathematical Institute, Slovak Academy of Sciences, \\ Štefánikova 49, 81473 Bratislava, Slovakia \\ jenca@mat.savba.sk
}

\begin{abstract}
A generalized channel is a completely positive map that preserves trace on a given subspace. We find conditions under which a generalized channel with respect to a positively generated subspace $J$ is an extreme point in the set of all such generalized channels. As a special case, this yields extremality conditions for quantum protocols. In particular, we obtain new extremality conditions for quantum 1-testers with 2 outcomes, which correspond to yes/no measurements on the set of quantum channels.
\end{abstract}

\section{Introduction}

Generalized channels with respect to a positively generated subspace $J$ of a finite-dimensional $C^{*}$-algebra $\mathcal{A}$ were introduced in 9 . These are completely positive maps $\mathcal{A} \rightarrow \mathcal{B}$ that preserve trace on $J$. If restricted to $J$, a generalized channel defines a channel on $J$, that is a completely positive trace preserving map $J \rightarrow \mathcal{B}$. Conversely, any channel on $J$ can be extended to a generalized channel. Since, in general, there are many generalized channels restricting to the same map on $J$, a channel on $J$ can be viewed as an equivalence class of generalized channels.

The motivation for the study of generalized channels comes from the description of quantum protocols by so-called generalized quantum instruments, [4, 7] (or quantum strategies [8]). These include quantum combs [1] describing quantum networks, and quantum testers 2 that describe measurements on combs. It can be seen [9] that all generalized quantum instruments are (multiples of) generalized channels with respect to certain subspaces that are given by the Choi isomorphism between completely positive maps and positive elements in tensor products.

A particular case of a generalized channel is a generalized POVM, which describes a measurement on the intersection of $J$ and the state space, in the same way that a positive operator valued measure (POVM) describes a usual measurement. Here, a measurement on a convex subset $K$ of the state space is naturally defined as an affine map from $K$ to the set of probability measures on

\footnotetext{
*Supported by by the grants VEGA 2/0032/09 and meta-QUTE ITMS 26240120022.
} 
the set of outcomes. But unlike the POVM, the generalized POVM describing a given measurement is in general not unique, so that again, measurements are equivalence classes of generalized POVMs.

If the subspace $J$ contains a positive invertible element, the set of generalized channels with respect to $J$ is a compact convex set, see [9, Proposition 6]. Hence any element of this set is a convex combination of its extreme points. Moreover, many optimalization problems consist in maximizing a convex function over the set of generalized channels, and this maximum is attained at an extreme point. Therefore, it is important to characterize extremal generalized channels. For generalized quantum instruments, this was done recently in [7.

In the present paper, we obtain a set of conditions characterizing extremal generalized channels with respect to $J$. These conditions are given in terms of the Choi representation, the Kraus representation and the conjugate map of $\Phi$. In the case of generalized quantum instruments, we get extremality conditions different from the ones obtained in 7. Moreover, we obtain conditions for extremality in the class of generalized POVMs for projection valued measures. In particular, this leads to a new characterization of extremal 1-testers with two outcomes.

\section{Preliminaries}

Let $\mathcal{A}$ be a finite dimensional $C^{*}$-algebra. Then $\mathcal{A}$ is isomorphic to a direct sum of matrix algebras, that is, there are finite dimensional Hilbert spaces $\mathcal{H}_{1}, \ldots \mathcal{H}_{n}$, such that

$$
\mathcal{A} \equiv \bigoplus_{j} B\left(\mathcal{H}_{j}\right)
$$

Below we always assume that $\mathcal{A}$ is equal to this direct sum. Let $s_{1}, \ldots s_{l}$ be the minimal central projections in $\mathcal{A}$ and let us fix an orthonormal basis $|i\rangle$, $i=1, \ldots, N$ of $\mathcal{H}=\oplus_{j} \mathcal{H}_{j}$, such that each $|i\rangle\langle i|$ commutes with all $s_{j}$. Then $\mathcal{A}$ is identified with the subalgebra of block-diagonal matrices in the matrix algebra $M_{N}(\mathbb{C}) \equiv B(\mathcal{H})$. The identity in $\mathcal{A}$ will be denoted by $I_{\mathcal{A}}$.

We fix a trace $\operatorname{Tr}_{\mathcal{A}}$ on $\mathcal{A}$ to be the restriction of the $\operatorname{trace}^{\operatorname{Tr}_{\mathcal{H}}}$ in $B(\mathcal{H})$, we omit the subscript $\mathcal{A}$ if no confusion is possible. The trace defines the HilbertSchmidt inner product in $\mathcal{A}$ by $\langle a, b\rangle=\operatorname{Tr} a^{*} b$. If $A \subset \mathcal{A}$ we denote by $A^{\perp}$ the orthogonal complement of $A$ with respect to $\langle\cdot, \cdot\rangle$. Let $E_{\mathcal{A}}: B(\mathcal{H}) \rightarrow \mathcal{A}$ be defined as

$$
E_{\mathcal{A}}(a)=\sum_{i} s_{i} a s_{i}, \quad a \in B(\mathcal{H}) .
$$

Then $\operatorname{Tr} a b=\operatorname{Tr} E_{\mathcal{A}}(a) b$ for all $a \in B(\mathcal{H}), b \in \mathcal{A}$ and $E_{\mathcal{A}}$ is the trace-preserving conditional expectation onto $\mathcal{A}$.

If $\mathcal{B}$ is another $C^{*}$ algebra, then $\operatorname{Tr}_{\mathcal{A}}^{\mathcal{A} \otimes \mathcal{B}}$ will denote the partial trace on the tensor product $\mathcal{A} \otimes \mathcal{B}, \operatorname{Tr}_{\mathcal{A}}^{\mathcal{A} \otimes \mathcal{B}}(a \otimes b)=\operatorname{Tr}(a) b$. If the input space is clear, we will denote the partial trace just by $\operatorname{Tr}_{\mathcal{A}}$.

For $a \in \mathcal{A}$, we denote by $a^{T}$ the transpose of $a$. Note that $\operatorname{Tr}_{\mathcal{A}}^{\mathcal{A} \otimes \mathcal{B}}\left(x^{T}\right)=$ $\left(\operatorname{Tr}_{\mathcal{A}}^{\mathcal{A} \otimes \mathcal{B}} x\right)^{T}$ for $x \in \mathcal{A} \otimes \mathcal{B}$. If $A \subset \mathcal{A}$, then $A^{T}=\left\{a^{T}, a \in A\right\}$. 
We denote by $\mathcal{A}^{+}$the convex cone of positive elements in $\mathcal{A}$ and $\mathfrak{S}(\mathcal{A})$ the set of states on $\mathcal{A}$, which will be identified with the set of density operators in $\mathcal{A}$, that is, elements $\rho \in \mathcal{A}^{+}$with $\operatorname{Tr} \rho=1$. For $a \in \mathcal{A}^{+}$, the projection onto the support of $a$ will be denoted by $\operatorname{supp}(a)$. If $p \in \mathcal{A}$ is a projection, we denote $\mathcal{A}_{p}=p \mathcal{A} p$.

Let $L \subseteq \mathcal{A}$ be a linear subspace, then $L$ is self-adjoint if $a^{*} \in L$ whenever $a \in L$. If $L$ is generated by positive elements, then we say that $L$ is positively generated. It is clear that $L$ is self-adjoint in this case.

\subsection{Completely positive maps, channels and generalized channels}

Let $\mathcal{A} \subseteq B(\mathcal{H}), \mathcal{B} \subseteq B(\mathcal{K})$ be finite dimensional $C^{*}$ algebras and let $J \subseteq \mathcal{A}$ be a positively generated subspace of $\mathcal{A}$. A linear map $\Xi: J \rightarrow \mathcal{B}$ is positive if $\Xi$ maps $J \cap \mathcal{A}^{+}$into $\mathcal{B}^{+}$and it is completely positive if the map

$$
\Xi \otimes i d_{\mathcal{H}_{0}}: J \otimes B\left(\mathcal{H}_{0}\right) \rightarrow \mathcal{B} \otimes B\left(\mathcal{H}_{0}\right)
$$

is positive for any finite dimensional Hilbert space $\mathcal{H}_{0}$. In this case, $\Xi$ extends to a completely positive map $\Phi: \mathcal{A} \rightarrow \mathcal{B}[9$.

Let $\Phi: \mathcal{A} \rightarrow \mathcal{B}$ be a linear map. Suppose first that $\mathcal{A}=B(\mathcal{H})$, then the Choi representation of $\Phi$ is defined as

$$
X_{\Phi}=\left(\Phi \otimes i d_{\mathcal{H}}\right)\left(\left|\psi_{\mathcal{H}}\right\rangle\left\langle\psi_{\mathcal{H}}\right|\right),
$$

where $\left|\psi_{\mathcal{H}}\right\rangle=\sum_{i}|i\rangle \otimes|i\rangle$, note that

$$
\left(a \otimes I_{\mathcal{H}}\right)\left|\psi_{\mathcal{H}}\right\rangle=\left(I_{\mathcal{H}} \otimes a^{T}\right)\left|\psi_{\mathcal{H}}\right\rangle, \quad a \in B(\mathcal{H}) .
$$

We have

$$
\Phi(a)=\operatorname{Tr}_{\mathcal{A}}\left[\left(I_{\mathcal{B}} \otimes a^{T}\right) X_{\Phi}\right], \quad a \in B(\mathcal{H})
$$

and $\Phi$ is completely positive if and only if $X_{\Phi} \geq 0$, [6]. Let now $\mathcal{A} \subset B(\mathcal{H})$ and let $\Phi^{\prime}=\Phi \circ E_{\mathcal{A}}$. Then $\Phi^{\prime}$ is an extension of $\Phi$ and it is completely positive if and only if $\Phi$ is. In this case, we define the Choi representation of $\Phi$ as

$$
X_{\Phi}:=X_{\Phi^{\prime}} .
$$

It is easy to see that $X_{\Phi} \in \mathcal{A} \otimes \mathcal{B}$ and that $\Phi$ is completely positive if and only if $X_{\Phi} \geq 0$. Moreover, (2) holds if $a \in \mathcal{A}$. If $\mathcal{A}=\bigoplus_{j} B\left(\mathcal{H}_{j}\right), \mathcal{B}=\bigoplus_{k} B\left(\mathcal{K}_{k}\right)$, then there are maps $\Phi_{j k}: B\left(\mathcal{H}_{j}\right) \rightarrow B\left(\mathcal{K}_{k}\right)$ such that

$$
\Phi(a)=\oplus_{k} \Phi_{j k}(a), \quad a \in B\left(\mathcal{H}_{j}\right)
$$

It is clear that $\Phi$ is completely positive if and only if $\Phi_{j k}$ are completely positive for all $j, k$.

A channel $\Xi: J \rightarrow \mathcal{B}$ is a trace preserving completely positive map. Clearly, a completely positive map $\Phi: \mathcal{A} \rightarrow \mathcal{B}$ is an extension of some channel $J \rightarrow \mathcal{B}$ if 
and only if $\Phi$ preserves trace on $J$, such a map is called a generalized channel. If $X_{\Phi} \in(\mathcal{B} \otimes \mathcal{A})^{+}$is the Choi representation of $\Phi: \mathcal{A} \rightarrow \mathcal{B}$, then $\Phi$ is a generalized channel if and only if

$$
\operatorname{Tr}_{\mathcal{B}} X \in\left(I_{\mathcal{A}}+\left(J^{T}\right)^{\perp}\right) \cap \mathcal{A}^{+}
$$

where $J^{\perp}$ denotes the orthogonal complement with respect to the HilbertSchmidt inner product. Let us denote the set of all generalized channels, resp. their Choi matrices, by $\mathcal{C}_{J}(\mathcal{A}, \mathcal{B})$. Clearly, if $J=\mathcal{A}$, we obtain the set of usual channels $\mathcal{A} \rightarrow \mathcal{B}$, this set will be denoted by $\mathcal{C}(\mathcal{A}, \mathcal{B})$.

Let $c \in \mathcal{A}$ and let us denote $\chi_{c}(a)=c a c^{*}$. Then $\chi_{c}: \mathcal{A} \rightarrow \mathcal{A}$ is clearly completely positive and it is a generalized channel if and only if

$$
c^{*} c \in\left(I_{\mathcal{A}}+J^{\perp}\right) \cap \mathcal{A}^{+},
$$

such generalized channels are called simple. The following is a slight modification of [9, Proposition 5].

Proposition 1 Let $\Phi: \mathcal{A} \rightarrow \mathcal{B}$ be a linear map. Then $\Phi$ is a generalized channel with respect to $J$ if and only if there is a simple generalized channel $\chi_{c}: \mathcal{A} \rightarrow \mathcal{A}$ and a channel $\Lambda: \mathcal{A} \rightarrow \mathcal{B}$, such that

$$
\Phi=\Lambda \circ \chi_{c} .
$$

Moreover, let $\Lambda_{q}$ be the restriction of $\Lambda$ to $\mathcal{A}_{q}, q=\operatorname{supp}\left(c c^{*}\right)$, then the pairs $(\Lambda, c)$ and $\left(\Lambda^{\prime}, c^{\prime}\right)$ define the same generalized channel if and only if there is a partial isometry $V$ with $V^{*} V=q, V V^{*}=q^{\prime}=\operatorname{supp}\left(c^{\prime} c^{*}\right)$ such that $c^{\prime}=V c$ and $\Lambda_{q^{\prime}}^{\prime}=\Lambda_{q} \circ \operatorname{Ad}_{V^{*}}$.

The decomposition $\Phi=\Lambda_{q} \circ \chi_{c}$ in the above proposition will be called minimal. Note that in terms of the Choi matrices, (4) has the form

$$
X_{\Phi}=\left(I_{\mathcal{B}} \otimes c^{T}\right) X_{\Lambda}\left(I_{\mathcal{B}} \otimes\left(c^{T}\right)^{*}\right) .
$$

Note also that $c^{*} c=\Phi^{*}\left(I_{\mathcal{B}}\right)=\left(\operatorname{Tr}_{\mathcal{B}} X_{\Phi}\right)^{T}$ whenever (4) holds.

Important examples of generalized channels will be treated in the sections below. In all examples, the subspace $J$ is of the form $J=S^{-1}\left(J_{0}\right)$, where $S: \mathcal{A} \rightarrow \mathcal{A}_{0}$ is a channel and $J_{0} \subseteq \mathcal{A}_{0}$ a subspace. Then 9 ]

$$
J^{\perp}=S^{*}\left(J_{0}^{\perp}\right),
$$

where $S^{*}: \mathcal{A}_{0} \rightarrow \mathcal{A}$ is the adjoint of $S$. Let $J_{0}$ be the one dimensional subspace generated by some $\rho_{0}=S(\rho)$ with invertible $\rho \in \mathfrak{S}(\mathcal{A})$, then

$$
J \cap \mathfrak{S}(\mathcal{A})=\left\{\sigma \in \mathfrak{S}(\mathcal{A}), S(\sigma)=\rho_{0}\right\}=: K_{S, \rho_{0}}
$$

is the equivalence class containing $\rho$ of the obvious equivalence relation on $\mathcal{A}$ defined by $S$.

Suppose moreover that the adjoint $S^{*}$ is an injective homomorphism, that is, $S^{*}\left(a_{0} b_{0}\right)=S^{*}\left(a_{0}\right) S^{*}\left(b_{0}\right)$ for all $a_{0}, b_{0} \in \mathcal{A}_{0}$ and $S^{*}\left(a_{0}\right)=0$ implies $a_{0}=0$ for $a_{0} \in \mathcal{A}_{0}$, note that this implies that $\rho_{0}$ is invertible. We will describe the simple generalized channels in this case. 
Lemma 1 Let $J$ be as above, then $d \in\left(I_{\mathcal{A}}+J^{\perp}\right) \cap \mathcal{A}^{+}$if and only if $d=S^{*}\left(b_{0}^{2}\right)$, where $b_{0}=\sigma_{0}^{1 / 2}\left(\sigma_{0}^{1 / 2} \rho_{0} \sigma_{0}^{1 / 2}\right)^{-1 / 2} \sigma_{0}^{1 / 2}$ for some $\sigma_{0} \in \mathfrak{S}\left(\mathcal{A}_{0}\right)$. Moreover, in this case,

$$
\chi_{d^{1 / 2}}\left(K_{S, \rho_{0}}\right) \subseteq K_{S, \sigma_{0}}
$$

Proof. By (5), we obtain

$$
\left(I_{\mathcal{A}}+J^{\perp}\right) \cap \mathcal{A}^{+}=\left(I_{\mathcal{A}}+S^{*}\left(\left\{\rho_{0}\right\}^{\perp}\right)\right) \cap \mathcal{A}^{+}=S^{*}\left(\left(I_{\mathcal{A}_{0}}+\left\{\rho_{0}\right\}^{\perp}\right) \cap \mathcal{A}_{0}^{+}\right)
$$

Moreover, let $b_{0} \in \mathcal{A}_{0}^{+}$and put $\sigma_{0}:=b_{0} \rho_{0} b_{0}$, then $b_{0}=\sigma_{0}^{1 / 2}\left(\sigma_{0}^{1 / 2} \rho_{0} \sigma_{0}^{1 / 2}\right)^{-1 / 2} \sigma_{0}^{1 / 2}$ is the unique positive solution of this equation. Clearly, $b_{0}^{2} \in I_{\mathcal{A}_{0}}+\left\{\rho_{0}\right\}^{\perp}$ if and only if $\sigma_{0} \in \mathfrak{S}\left(\mathcal{A}_{0}\right)$.

Let now $\chi_{d^{1 / 2}}$ be a simple generalized channel, so that $d^{1 / 2}=S^{*}\left(b_{0}\right)$. For $a \in \mathcal{A}, a_{0} \in \mathcal{A}_{0}$, we have

$$
\operatorname{Tr} S\left(\chi_{d^{1 / 2}}(a)\right) a_{0}=\operatorname{Tr} S^{*}\left(b_{0}\right) a S^{*}\left(b_{0}\right) S^{*}\left(a_{0}\right)=\operatorname{Tr} b_{0} S(a) b_{0} a_{0}
$$

so that $S\left(\chi_{d^{1 / 2}}(a)\right)=\chi_{b_{0}}(S(a))=\sigma_{0}$ if $S(a)=\rho_{0}$.

An example of this situation is contained in Section 2.1.1. We will show one more simple example.

Example 1 Let $\mathcal{D}_{n}$ denote the set of density matrices in the matrix algebra $M_{n}(\mathbb{C})$. Let $\lambda=\left(\lambda_{1}, \ldots, \lambda_{n}\right), \lambda_{i}>0, \sum_{i} \lambda_{i}=1$ and let $\operatorname{Diag}_{\lambda}$ be the set of density matrices $\rho=\left\{\rho_{i j}\right\}_{i, j} \in \mathcal{D}_{n}$ such that $\rho_{i i}=\lambda_{i}$ for all $i$. Let $S: M_{n}(\mathbb{C}) \rightarrow$ $\mathbb{C}^{n}$ be the map that maps every matrix onto the vector of its diagonal elements, that is,

$$
S(a)=(\langle 1, a 1\rangle, \ldots,\langle n, a n\rangle), \quad a \in M_{n}(\mathbb{C})
$$

where $|i\rangle$ denotes the standard basis of $\mathbb{C}^{n}$. Then $S$ is a channel and $\operatorname{Diag}_{\lambda}=$ $J_{\lambda} \cap \mathcal{D}_{n}=K_{S, \lambda}$ in the notation of (6), here $J_{\lambda}:=S^{-1}(\mathbb{C} \lambda)$. Moreover, it is easy to see that $S^{*}(x)=\sum_{i} x_{i}|i\rangle\langle i|$ for any $x \in \mathbb{C}^{n}$ is an isomorphism onto the commutative subalgebra generated by $|i\rangle\langle i|$. It follows that all simple generalized channels are obtained from elements of the form $c=U d^{1 / 2}$, where $U$ is a partial isometry and $d=\sum_{i} d_{i}|i\rangle\langle i|, d_{i}=\mu_{i} / \lambda_{i}$ for some probability vector $\mu=\left(\mu_{1}, \ldots, \mu_{n}\right)$. In particular, $\chi_{d^{1 / 2}}$ maps $\operatorname{Diag}_{\lambda}$ onto Diag . .

\subsubsection{Channels on channels}

Let $\mathcal{H}_{0}, \mathcal{H}_{1}$ be finite dimensional Hilbert spaces and let $\mathcal{C}\left(\mathcal{H}_{0}, \mathcal{H}_{1}\right)$ denote the set of Choi matrices of channels $B\left(\mathcal{H}_{0}\right) \rightarrow B\left(\mathcal{H}_{1}\right)$. Then

$$
\mathcal{C}\left(\mathcal{H}_{0}, \mathcal{H}_{1}\right)=\left\{X \in B\left(\mathcal{H}_{1} \otimes \mathcal{H}_{0}\right)^{+}, \operatorname{Tr}_{\mathcal{H}_{1}} X=I_{\mathcal{H}_{0}}\right\}
$$

Let $\mathcal{A}=B\left(\mathcal{H}_{1} \otimes \mathcal{H}_{0}\right)$ and let $J \subset \mathcal{A}$ be the subspace generated by $\mathcal{C}\left(\mathcal{H}_{0}, \mathcal{H}_{1}\right)$. Then $J=\operatorname{Tr}_{\mathcal{H}_{1}}^{-1}\left(\mathbb{C} I_{\mathcal{H}_{0}}\right)$ and $\mathcal{C}\left(\mathcal{H}_{0}, \mathcal{H}_{1}\right)=J \cap \operatorname{dim}\left(\mathcal{H}_{0}\right) \mathfrak{S}(\mathcal{A})$, hence $\mathcal{C}\left(\mathcal{H}_{0}, \mathcal{H}_{1}\right)$ is a constant multiple of a set of the form (6). Moreover,

$$
S^{*}\left(a_{0}\right)=\operatorname{Tr}_{\mathcal{H}_{1}}^{*}\left(a_{0}\right)=I_{\mathcal{H}_{1}} \otimes a_{0}, \quad a_{0} \in B\left(\mathcal{H}_{0}\right),
$$


hence we may apply Lemma 1 Note also that $J^{T}=J$ and

$$
J^{\perp}=I_{\mathcal{H}_{1}} \otimes \mathcal{T}\left(\mathcal{H}_{0}\right),
$$

where $\mathcal{T}\left(\mathcal{H}_{0}\right)$ is the subspace of traceless elements in $B\left(\mathcal{H}_{0}\right)$.

Let $\mathcal{B}$ be a finite dimensional $C^{*}$ algebra. Let us denote by $\mathcal{C}\left(\mathcal{H}_{0}, \mathcal{H}_{1}, \mathcal{B}\right)$ the set of Choi matrices of completely positive maps $\mathcal{A} \rightarrow \mathcal{B}$ that map $\mathcal{C}\left(\mathcal{H}_{0}, \mathcal{H}_{1}\right)$ into the state space $\mathfrak{S}(\mathcal{B})$. Then $\mathcal{C}\left(\mathcal{H}_{0}, \mathcal{H}_{1}, \mathcal{B}\right)=\operatorname{dim}\left(\mathcal{H}_{0}\right)^{-1} \mathcal{C}_{J}(\mathcal{A}, \mathcal{B})$. It follows that $X \in(\mathcal{B} \otimes \mathcal{A})^{+}$is in $\mathcal{C}\left(\mathcal{H}_{0}, \mathcal{H}_{1}, \mathcal{B}\right)$ if and only if

$$
\operatorname{Tr}_{\mathcal{B}} X=I_{\mathcal{H}_{1}} \otimes \omega, \quad \omega \in \mathfrak{S}\left(\mathcal{H}_{0}\right)
$$

Let $X=X_{\Phi} \in \mathcal{C}\left(\mathcal{H}_{0}, \mathcal{H}_{1}, \mathcal{B}\right)$ and let $\operatorname{Tr}_{\mathcal{B}} X=I_{\mathcal{H}_{1}} \otimes \omega, \omega \in B\left(\mathcal{H}_{0}\right)$. Let

$$
\Phi=\Lambda_{q} \circ \chi_{I \otimes c}, \quad c^{*} c=\omega, \quad q=\operatorname{supp}\left(c c^{*}\right)
$$

be a minimal decomposition. Then for any $X_{\mathcal{E}} \in \mathcal{C}\left(\mathcal{H}_{0}, \mathcal{H}_{1}\right)$, we have

$$
\chi_{I \otimes c}\left(X_{\mathcal{E}}\right)=\chi_{I \otimes c} \circ\left(\mathcal{E} \otimes i d_{\mathcal{H}_{0}}\right)\left(\left|\psi_{\mathcal{H}_{0}}\right\rangle\left\langle\psi_{\mathcal{H}_{0}}\right|\right)=\left(\mathcal{E} \otimes i d_{q \mathcal{H}_{0}}\right)(\rho)
$$

where $\rho=\chi_{I \otimes c}\left(\left|\psi_{\mathcal{H}_{0}}\right\rangle\left\langle\psi_{\mathcal{H}_{0}}\right|\right) \in B\left(\mathcal{H}_{0} \otimes q \mathcal{H}_{0}\right)$ is a pure state, such that $\operatorname{Tr}_{2} \rho=$ $\omega^{T}$. This leads to the following implementation for $\Phi$ ([9, Theorem 4]):

Proposition 2 There is a Hilbert space $\mathcal{H}_{A}$, a pure state $\rho \in \mathcal{H}_{0} \otimes \mathcal{H}_{A}$ and a channel $\Lambda: B\left(\mathcal{H}_{1} \otimes \mathcal{H}_{A}\right) \rightarrow \mathcal{B}$ such that

$$
\Phi\left(X_{\mathcal{E}}\right)=\Lambda \circ\left(\mathcal{E} \otimes i d_{\mathcal{H}_{A}}\right)(\rho),
$$

for all channels $\mathcal{E}: B\left(\mathcal{H}_{0}\right) \rightarrow B\left(\mathcal{H}_{1}\right)$. Conversely, any map of the form (7) defines an element in $\mathcal{C}\left(\mathcal{H}_{0}, \mathcal{H}_{1}, \mathcal{B}\right)$.

The triple $\left(\mathcal{H}_{A}, \rho, \Lambda\right)$ as above will be called a representation of $X$. Moreover, if $\operatorname{dim}\left(\mathcal{H}_{A}\right)=\operatorname{rank}\left(\operatorname{Tr}_{\mathcal{H}_{A}} \rho\right)$, then the triple $\left(\mathcal{H}_{A}, \rho, \Lambda\right)$ will be called a minimal representation of $X$. If $\left(\mathcal{H}_{A}, \rho, \Lambda\right)$ and $\left(\mathcal{H}_{B}, \rho^{\prime}, \Lambda^{\prime}\right)$ are two minimal representations of $X$, then there is a unitary operator $U: \mathcal{H}_{A} \rightarrow \mathcal{H}_{B}$ such that $\rho^{\prime}=(I \otimes U) \rho\left(I \otimes U^{*}\right)$ and $\Lambda^{\prime}=\Lambda \circ A d_{I \otimes U^{*}}$.

\subsubsection{Quantum supermaps and generalized quantum instruments}

Let $\mathcal{B}_{0}, \mathcal{B}_{1}, \ldots$, be a sequence of finite dimensional $C^{*}$-algebras and let $\mathcal{A}_{n}:=$ $\mathcal{B}_{n} \otimes \cdots \otimes \mathcal{B}_{0}$. For $n \in \mathbb{N}$, the sets $\mathcal{C}\left(\mathcal{B}_{0}, \ldots, \mathcal{B}_{n}\right)$ are defined as follows:

Let $\mathcal{C}\left(\mathcal{B}_{0}, \mathcal{B}_{1}\right)$ be the set of the Choi matrices of channels $\mathcal{B}_{0} \rightarrow \mathcal{B}_{1}$. For $n>1, \mathcal{C}\left(\mathcal{B}_{0}, \ldots, \mathcal{B}_{n}\right)$ is defined as the set of Choi matrices of completely positive maps $\mathcal{A}_{n-1} \rightarrow \mathcal{B}_{n}$, that map $\mathcal{C}\left(\mathcal{B}_{0}, \ldots, \mathcal{B}_{n-1}\right)$ into $\mathfrak{S}\left(\mathcal{B}_{n}\right)$. Such maps are called quantum supermaps, note that this definition is different from the definition of a quantum supermap given in [3. It is clear that $\mathcal{C}\left(\mathcal{H}_{0}, \mathcal{H}_{1}, \mathcal{B}\right)$ from Section 2.1 .1 is a set of quantum supermaps for $n=2$. 


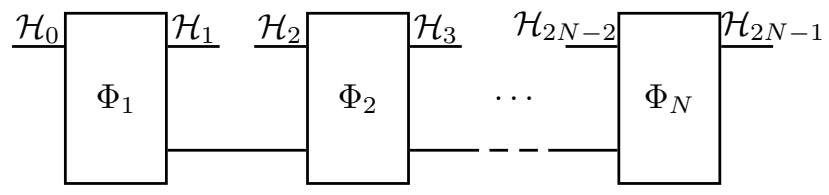

Figure 1: A deterministic quantum $N$-comb

The set $\mathcal{C}\left(\mathcal{B}_{0}, \ldots, \mathcal{B}_{n}\right)$ is a constant multiple of a set of generalized channels, more precisely,

$$
\mathcal{C}\left(\mathcal{B}_{0}, \ldots, \mathcal{B}_{n}\right)=J_{n} \cap c_{n} \mathfrak{S}\left(\mathcal{A}_{n}\right)=\frac{1}{c_{n-1}} \mathcal{C}_{J_{n-1}}\left(\mathcal{A}_{n-1}, \mathcal{B}_{n}\right)
$$

where $c_{n}:=\Pi_{l=0}^{\left\lfloor\frac{n-1}{2}\right\rfloor} \operatorname{Tr}\left(I_{\mathcal{B}_{n-1-2 l}}\right)$ and $J_{n}:=J_{n}\left(\mathcal{B}_{0}, \ldots, \mathcal{B}_{n}\right)$ denotes the subspace in $\mathcal{A}_{n}$ generated by $\mathcal{C}\left(\mathcal{B}_{0}, \ldots, \mathcal{B}_{n}\right)$. The subspaces $J_{n}$ are clearly positively generated and obtained as follows: Let $S_{n}: \mathcal{A}_{n} \rightarrow \mathcal{A}_{n-1}$ denote the partial trace $\operatorname{Tr}_{\mathcal{B}_{n}}^{\mathcal{A}_{n}}, n=1,2, \ldots$, then we have

$$
\begin{aligned}
J_{2 k-1} & =S_{2 k-1}^{-1}\left(S_{2 k-2}^{*}\left(S_{2 k-3}^{-1}\left(\ldots S_{1}^{-1}\left(\mathbb{C} I_{\mathcal{B}_{0}}\right) \ldots\right)\right)\right) \\
J_{2 k} & =S_{2 k}^{-1}\left(S_{2 k-1}^{*}\left(S_{2 k-2}^{-1}\left(\ldots S_{1}^{*}\left(\mathcal{B}_{0}\right) \ldots\right)\right)\right)
\end{aligned}
$$

There is another way to characterize the elements in $\mathcal{C}\left(\mathcal{B}_{0}, \ldots, \mathcal{B}_{n}\right)$ : Let $k:=$ $\left\lfloor\frac{n}{2}\right\rfloor$. Then $X \in \mathcal{C}\left(\mathcal{B}_{0}, \ldots, \mathcal{B}_{n}\right)$ if and only if there are positive elements $Y^{(m)} \in$ $\mathcal{A}_{n-2 m}$ for $m=0, \ldots, k$, such that

$$
\operatorname{Tr}_{\mathcal{B}_{n-2 m}} Y^{(m)}=I_{\mathcal{B}_{n-2 m-1}} \otimes Y^{(m+1)}, m=0, \ldots, k-1
$$

$Y^{(0)}:=X, Y^{(k)} \in \mathcal{C}\left(\mathcal{B}_{0}, \mathcal{B}_{1}\right)$ if $n=2 k+1$ and $Y^{(k)} \in \mathfrak{S}\left(\mathcal{B}_{0}\right)$ if $n=2 k$.

Let $\mathcal{H}_{0}, \mathcal{H}_{1}, \ldots$ be finite dimensional Hilbert spaces. As it was pointed out in [9], the elements of $\mathcal{C}\left(\mathcal{H}_{0}, \ldots, \mathcal{H}_{2 N-1}\right)$ are precisely the (deterministic) quantum $N$-combs on $\left(\mathcal{H}_{0}, \ldots, \mathcal{H}_{2 N-1}\right)$ [4, which are the Choi matrices of completely positive maps used in description of quantum networks. More precisely, quantum 1-combs are defined as the elements of $\mathcal{C}\left(\mathcal{H}_{0}, \mathcal{H}_{1}\right)$ and for $N>1$, quantum $N$-combs are completely positive maps that map the $N-1$ combs on $\left(\mathcal{H}_{1}, \ldots, \mathcal{H}_{2 N-1}\right)$ to $\mathcal{C}\left(\mathcal{H}_{0}, \mathcal{H}_{2 N-1}\right)$. Quantum $N$-combs can be represented by memory channels, given by a sequence of $N$ channels connected by an ancilla, see Fig. 1

Another special case is the set of quantum $N$-testers (with $m$ outcomes), which describe measurements on quantum $N$-combs. These are precisely the elements of the set $\mathcal{C}\left(\mathcal{H}_{0}, \ldots, \mathcal{H}_{2 N-1}, \mathbb{C}^{m}\right)$. More generally, if some of the channels in the sequence representing the $N$-comb are replaced by instruments or POVMs, we obtain the set of generalized quantum instruments, 44, 7]. These are described by probabilistic $N$-combs, which are positive operators $X_{i}$, such that $\sum_{i} X_{i}$ is a (deterministic) quantum $N$-comb. 


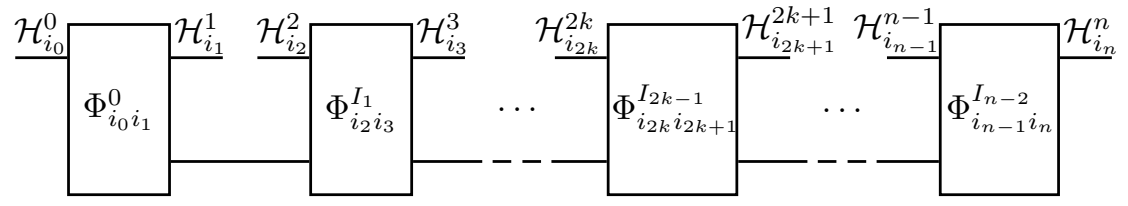

Figure 2: Representation of a quantum supermap, $n=2 N-1, I=\left(i_{0}, \ldots, i_{n}\right)$

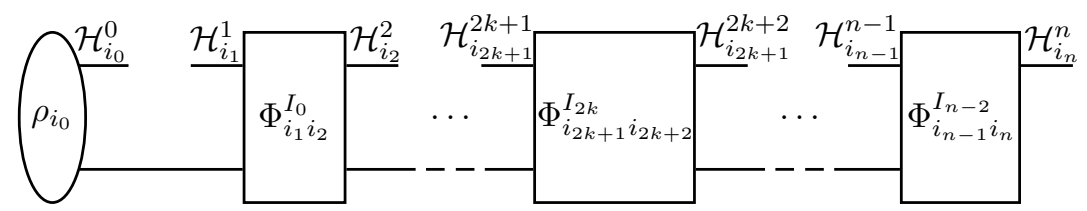

Figure 3: Representation of a quantum supermap, $n=2 N, I=\left(i_{0}, \ldots, i_{n}\right)$

If the matrix algebras $B\left(\mathcal{H}_{n}\right)$ are replaced by finite dimensional $C^{*}$-algebras, it can be seen that the set of quantum supermaps with $n=2 N-1$ correspond to conditional quantum combs [5], which describe quantum protocols where classical inputs and outputs are allowed. Let $\mathcal{B}_{j}=\bigoplus_{k=1}^{n_{j}} B\left(\mathcal{H}_{k}^{j}\right), j=0, \ldots, n$, then any element in $\mathcal{C}\left(\mathcal{B}_{0}, \ldots, \mathcal{B}_{2 N-1}\right)$ is represented by a sequence of networks as in Fig. 2. The elements of the sequence are labelled by multiindices $I=$ $\left(i_{0}, \ldots, i_{n}\right), i_{j} \in\left\{1, \ldots, n_{j}\right\}$, representing the classical inputs and outputs, $I_{j}=$ $\left(i_{0}, \ldots, i_{j}\right)$ and $\Phi^{0}: \mathcal{B}_{0} \rightarrow \mathcal{B}_{1}, \Phi^{I_{2 k-1}}: \mathcal{B}_{2 k} \rightarrow \mathcal{B}_{2 k+1}, k=1, \ldots, N-1$ are channels. For $n=2 N$, the elements in $\mathcal{C}\left(\mathcal{B}_{0}, \ldots, \mathcal{B}_{n}\right)$ are represented similarly, see Fig. 3, here $\rho_{0}=\oplus_{i_{0}} \rho_{i_{0}} \in \mathfrak{S}\left(\mathcal{B}_{0}\right)$ and $\Phi^{I_{2 k}}: \mathcal{B}_{2 k+1} \rightarrow \mathcal{B}_{2 k+2}$ are channels, $k=0, \ldots, N-1$, see [9, Theorem 8].

\section{$3 \quad$ Extremal generalized channels}

Let $J \subseteq \mathcal{A}$ be a positively generated subspace. Let us denote

$$
L:=\left\{X \in \mathcal{B} \otimes \mathcal{A}, \operatorname{Tr}_{\mathcal{B}}(X) \in I_{\mathcal{A}}+\left(J^{T}\right)^{\perp}\right\},
$$

then $L$ is an affine subspace in $\mathcal{B} \otimes \mathcal{A}$ and we have

$$
\operatorname{Lin}(L):=\{X-Y: X, Y \in L\}=\left\{X \in \mathcal{B} \otimes \mathcal{A}, \operatorname{Tr}_{\mathcal{B}}(X) \in\left(J^{T}\right)^{\perp}\right\}
$$

It is easy to see that

$$
\mathcal{C}_{J}(\mathcal{A}, \mathcal{B})=(\mathcal{B} \otimes \mathcal{A})^{+} \cap L,
$$

It is also clear that the faces in $\mathcal{C}_{J}(\mathcal{A}, \mathcal{B})$ are precisely the sets of the form

$$
F=(\mathcal{B} \otimes \mathcal{A})_{P}^{+} \cap L
$$


for a projection $P \in \mathcal{B} \otimes \mathcal{A}$. Indeed, it is quite clear that any such subset is a face. Conversely, let $F \subset \mathcal{C}_{J}(\mathcal{A}, \mathcal{B})$ be a face and let $X \in F$ be an element with maximal support, $P=\operatorname{supp}(X)$. Then $F \subseteq G:=(\mathcal{B} \otimes \mathcal{A})_{P}^{+} \cap L$. Since both $F$ and $G$ are faces in $\mathcal{C}_{J}(\mathcal{A}, \mathcal{B})$ and $X \in F \cap \operatorname{ri}(G)$, where $r i(G)$ denotes the relative interior of $G$, it follows by [10, Theorem, 18.1] that $F=G$.

Theorem 1 Let $X \in \mathcal{C}_{J}(\mathcal{A}, \mathcal{B})$ and let $P=\operatorname{supp}(X)$. Then $X$ is extremal if and only if

$$
(\mathcal{B} \otimes \mathcal{A})_{P} \cap \operatorname{Lin}(L)=\{0\}
$$

Proof. Suppose that $X=\frac{1}{2}\left(X_{1}+X_{2}\right)$ for some $X_{1}, X_{2} \in \mathcal{C}_{J}(\mathcal{A}, \mathcal{B})$. Then $X_{1}, X_{2} \in(\mathcal{B} \otimes \mathcal{A})_{P}^{+} \cap L$ so that $X_{1}-X_{2} \in(\mathcal{A} \otimes \mathcal{B})_{P} \cap \operatorname{Lin}(L)$. Conversely, let $Y \in(\mathcal{B} \otimes \mathcal{A})_{P} \cap \operatorname{Lin}(L)$, then also $Y+Y^{*} \in(\mathcal{B} \otimes \mathcal{A})_{P} \cap \operatorname{Lin}(L)$, hence we may suppose that $Y$ is self adjoint. Then there exists some $t>0$ such that $Z_{ \pm}:=X \pm t Y \in \mathcal{C}_{J}(\mathcal{A}, \mathcal{B})$, moreover, $X=\frac{1}{2}\left(Z_{+}+Z_{-}\right)$.

Corollary 1 Let $X$ be an extreme point in $\mathcal{C}_{J}(\mathcal{A}, \mathcal{B})$. Let $P=\operatorname{supp}(X)$ and let $p=\operatorname{supp}\left(\operatorname{Tr}_{\mathcal{B}} X\right)^{T}$. Then

$$
\operatorname{dim}(\mathcal{B} \otimes \mathcal{A})_{P} \leq \operatorname{dim}(p J p)
$$

Proof. Note that we have

$$
\operatorname{Tr} X\left(I_{\mathcal{B} \otimes \mathcal{A}}-I_{\mathcal{B}} \otimes p^{T}\right)=\operatorname{Tr} X\left(I_{\mathcal{B}} \otimes\left(I_{\mathcal{A}}-p^{T}\right)\right)=\operatorname{Tr}\left(I_{\mathcal{A}}-p^{T}\right) \operatorname{Tr}_{\mathcal{B}} X=0,
$$

so that $P \leq I_{\mathcal{B}} \otimes p^{T}$. Let $L_{p^{T}}:=L \cap\left(\mathcal{B} \otimes \mathcal{A}_{p^{T}}\right)$, then

$$
(\mathcal{B} \otimes \mathcal{A})_{P} \cap \operatorname{Lin}\left(L_{p^{T}}\right)=(\mathcal{B} \otimes \mathcal{A})_{P} \cap \operatorname{Lin}(L)
$$

Moreover, since $\left(J^{T}\right)^{\perp} \cap \mathcal{A}_{p^{T}}=\left((p J p)^{T}\right)^{\perp} \cap \mathcal{A}_{p^{T}}$,

$$
\operatorname{Lin}\left(L_{p^{T}}\right)=\left\{Y \in \mathcal{B} \otimes \mathcal{A}_{p^{T}}, \operatorname{Tr}_{\mathcal{B}} Y \in\left((p J p)^{T}\right)^{\perp} \cap \mathcal{A}_{p^{T}}\right\} .
$$

Suppose that $X$ is an extreme point, then $(\mathcal{B} \otimes \mathcal{A})_{P} \cap \operatorname{Lin}\left(L_{p^{T}}\right)=\{0\}$, so that, by applying the orthogonal complements in $\mathcal{B} \otimes \mathcal{A}_{p^{T}}$,

$$
(\mathcal{B} \otimes \mathcal{A})_{P}^{\perp} \vee \operatorname{Lin}\left(L_{p^{T}}\right)^{\perp}=\mathcal{B} \otimes \mathcal{A}_{p^{T}}
$$

It follows that we must have $\operatorname{dim}\left((\mathcal{B} \otimes \mathcal{A})_{P}\right) \leq \operatorname{dim}\left(\operatorname{Lin}\left(L_{p^{T}}\right)^{\perp}\right)$. The statement now follows from $\operatorname{Lin}\left(L_{p^{T}}\right)^{\perp}=I_{\mathcal{B}} \otimes(p J p)^{T}$, by (5) .

Let $\Phi=\Lambda_{q} \circ \chi_{c}$ be a minimal decomposition of $\Phi, p=\operatorname{supp}\left(c^{*} c\right), q=$ $\operatorname{supp}\left(c c^{*}\right)$. The next Proposition gives the extremality condition in terms of the channel $\Lambda_{q}$. Note that as a channel, $\Lambda_{q}$ is also a generalized channel with respect to any subspace in $\mathcal{A}_{q}$.

Theorem $2 \Phi$ is extremal in $\mathcal{C}_{J}(\mathcal{A}, \mathcal{B})$ if and only if $\Lambda_{q}$ is extremal in $\mathcal{C}_{c J c^{*}}\left(\mathcal{A}_{q}, \mathcal{B}\right)$ 
Proof. Suppose $\Lambda_{q}=\frac{1}{2}\left(\Psi_{1}+\Psi_{2}\right)$ for some $\Psi_{1} \neq \Psi_{2} \in \mathcal{C}_{c J c^{*}}\left(\mathcal{A}_{q}, \mathcal{B}\right)$. Put $\Phi_{i}=\Psi_{i} \circ \chi_{c}$, then $\Phi_{i}$ is completely positive and for any $a \in J$

$$
\operatorname{Tr} \Phi_{i}(a)=\operatorname{Tr} \Psi_{i}\left(c a c^{*}\right)=\operatorname{Tr} c a c^{*}=\operatorname{Tr} a
$$

so that $\Phi_{i} \in \mathcal{C}_{J}(\mathcal{A}, \mathcal{B})$. Clearly, $\Phi=\frac{1}{2}\left(\Phi_{1}+\Phi_{2}\right)$ and $\Phi_{1} \neq \Phi_{2}$.

Conversely, suppose that $\Phi=\frac{1}{2}\left(\Phi_{1}+\Phi_{2}\right), \Phi_{1} \neq \Phi_{2} \in \mathcal{C}_{J}(\mathcal{A}, \mathcal{B})$. Then $\Phi_{i}$ are decomposed as $\Phi_{i}=\Lambda_{i} \circ \chi_{c_{i}}$ for some channels $\Lambda_{i}: \mathcal{A} \rightarrow \mathcal{B}$ and $\Phi_{i}^{*}\left(I_{\mathcal{B}}\right)=c_{i}^{*} c_{i}$. Clearly, $c^{*} c=\frac{1}{2}\left(c_{1}^{*} c_{1}+c_{2}^{*} c_{2}\right)$, so that $\operatorname{supp}\left(c_{i}^{*} c_{i}\right) \leq \operatorname{supp}\left(c^{*} c\right)=p$. Put $\Psi_{i}=\Phi_{i}$ 。 $\chi_{c^{-1}}$, where $c^{-1}=\left(c^{*} c\right)^{-1 / 2} U^{*}$ for the polar decomposition $c=U\left(c^{*} c\right)^{1 / 2}$, the inverse being taken on the support of $c^{*} c$. Then $\Phi_{i}$ is a well defined completely positive map $\mathcal{A}_{q} \rightarrow \mathcal{B}$. Moreover,

$$
\operatorname{Tr} \Psi_{i}\left(c y c^{*}\right)=\operatorname{Tr} \Phi_{i}(y)=\operatorname{Tr} y, \quad y \in J
$$

so that $\Psi_{i} \in \mathcal{C}_{c J c^{*}}\left(\mathcal{A}_{q}, \mathcal{B}\right)$. We also have

$$
\frac{1}{2}\left(\Psi_{1}+\Psi_{2}\right) \circ \chi_{c}=\frac{1}{2}\left(\Phi_{1}+\Phi_{2}\right) \circ \chi_{c^{-1}} \circ \chi_{c}=\Phi \circ \chi_{p}=\Phi=\Lambda_{q} \circ \chi_{c}
$$

By uniqueness, $\Lambda_{q}=\frac{1}{2}\left(\Psi_{1}+\Psi_{2}\right)$.

\subsection{Extremality conditions for Kraus operators}

Let $\mathcal{A}=\oplus_{i} B\left(\mathcal{H}_{i}\right), \mathcal{B}=\oplus_{j} B\left(\mathcal{K}_{j}\right), \mathcal{H}=\oplus_{i} \mathcal{H}_{i}, \mathcal{K}=\oplus_{j} \mathcal{K}_{j}$. Let $\left\{\left|v_{k}\right\rangle\right\}_{k}$ be a set of vectors in $\mathcal{K} \otimes \mathcal{H}$, such that

$$
X_{\Phi}=\sum_{k}\left|v_{k}\right\rangle\left\langle v_{k}\right|
$$

and for each $i, j$, there is a set $I(i, j)$ of indices such that $\left|v^{k}\right\rangle \in \mathcal{K}_{i} \otimes \mathcal{H}_{j}$ for $k \in I(i, j)$. For example, we can choose $\left|v_{k}\right\rangle$ to be eigenvectors of $X_{\Phi}$. It is quite clear that the vectors $\left|v_{k}\right\rangle$ span $P(\mathcal{K} \otimes \mathcal{H})$, where $P=\operatorname{supp}\left(X_{\Phi}\right)$.

For each $j$, let $\left\{\left|\alpha_{m}^{j}\right\rangle\right\}_{m}$ be an ONB in $\mathcal{H}_{j}$. Then for each $k \in I(i, j)$ there are some vectors $\left|v_{m}^{k}\right\rangle \in \mathcal{K}_{i}$, such that $\left|v^{k}\right\rangle=\sum_{m}\left|v_{m}^{k}\right\rangle \otimes\left|\alpha_{m}^{j}\right\rangle$. Moreover, there is a one-to-one correspondence between vectors in $\mathcal{K}_{i} \otimes \mathcal{H}_{j}$ and linear maps $\mathcal{H}_{j} \rightarrow \mathcal{K}_{i}$, given by $|x\rangle \otimes|y\rangle \mapsto|x\rangle\langle y|$. In this correspondence,

$$
\left|v^{k}\right\rangle \mapsto V_{k}:=\sum_{m}\left|v_{m}^{k}\right\rangle\left\langle\alpha_{m}^{j}\right| .
$$

The map $\Phi$ has the form

$$
\Phi(a)=\sum_{k} V_{k} a V_{k}^{*}=\sum_{i, j} \sum_{k \in I(i, j)} V_{k} a V_{k}^{*}
$$

so that $V_{k}$ are Kraus operators of $\Phi$. 
Suppose that the vectors $\left\{\left|v_{k}\right\rangle\right\}_{k}$ are linearly independent, then the operators $V_{k}$ are linearly independent as well and (14) is a minimal Kraus representation of $\Phi$, 6. Conversely, any minimal Kraus representation is obtained in this way. It follows that $\left\{\left|v^{k}\right\rangle\left\langle v^{l}\right|, k, l \in I(i, j)\right\}_{i, j}$ is a basis of $(\mathcal{B} \otimes \mathcal{A})_{P}$, so that any element $D \in(\mathcal{B} \otimes \mathcal{A})_{P}$ can be uniquely expressed as

$$
D=\sum_{i, j} \sum_{k, l \in I(i, j)} d_{k, l}\left|v^{k}\right\rangle\left\langle v^{l}\right|
$$

Let us fix a basis $\left\{x_{1}, \ldots, x_{M}\right\}$ of $J^{\perp}$.

Corollary 2 Let $\Phi=\sum_{i, j} \sum_{k \in I(i, j)} V_{k} a V_{k}^{*}$ be a minimal Kraus representation. Then $\Phi$ is extremal if and only if the set

$$
\cup_{i, j}\left\{V_{k}^{*} V_{l}: k, l \in I(i, j)\right\} \cup\left\{x_{1}, \ldots, x_{M}\right\}
$$

is linearly independent.

Proof. Let $D \in(\mathcal{B} \otimes \mathcal{A})_{P}$ have the form (15), then

$$
\operatorname{Tr}_{\mathcal{B}} D=\sum_{i, j} \sum_{k, l \in I(i, j)} d_{k, l}\left(V_{k}^{*} V_{l}\right)^{T}
$$

By Theorem 1, $X$ is extremal if and only if

$$
\sum_{i, j} \sum_{k, l \in I(i, j)} d_{k, l} V_{k}^{*} V_{l} \in J^{\perp}
$$

for some coefficients $d_{k, l}$ implies that all $d_{k, l}=0$. This is exactly what we needed to prove.

Note that for ordinary channels, Corollary 2 gives the well known extremality condition obtained in [6], namely that the set $\left\{V_{k} V_{l}^{*}, k, l \in I(i, j), i, j\right\}$ is linearly independent.

Let now $\Phi=\Lambda_{q} \circ \chi_{c}$ be a minimal decomposition and let $\Lambda_{q}(a)=\sum_{k} W_{k} a W_{k}^{*}$ be a Kraus representation. As above, since $q \in \mathcal{A}$, we may suppose that for each $(i, j)$ there is some set of indices $J_{W}(i, j)$, such that for $k \in J_{W}(i, j)$, $W_{k}: \mathcal{H}_{j} \rightarrow \mathcal{K}_{i}$.

Corollary 3 Let $\Phi=\Lambda_{q} \circ \chi_{c}$ be a minimal decomposition and let $\Lambda_{q}(a)=$ $\sum_{k} W_{k} a W_{k}^{*}$ be a Kraus representation. Then $\Phi$ is extremal if and only if $\Lambda_{q}$ is an extremal channel and

$$
\operatorname{span}\left\{W_{k}^{*} W_{l}, k, l \in J_{W}(i, j), i, j\right\} \cap\left(c J c^{*}\right)^{\perp_{q}}=\{0\}
$$

where $L^{\perp_{q}}=L^{\perp} \cap \mathcal{A}_{q}$ for any subspace $L \subseteq \mathcal{A}_{q}$. 
Proof. By Theorem 2, $\Phi$ is extremal if and only if $\Lambda_{q}$ is an extremal generalized channel with respect to $c J c^{*}$. Let $\Lambda_{q}(a)=\sum_{i, j} \sum_{k \in J_{U}(i, j)} U_{k} a U_{k}^{*}$ be a minimal Kraus representation. Then Corollary 2 can be reformulated as follows: $\Lambda_{q}$ is extremal in $\mathcal{C}_{c J c^{*}}\left(\mathcal{A}_{q}, \mathcal{B}\right)$ if and only if the set $\left\{U_{k}^{*} U_{l}, k, l \in J_{U}(i, j), i, j\right\}$ is linearly independent (equivalently, $\Lambda_{q}$ is an extremal channel) and

$$
\operatorname{span}\left\{U_{k}^{*} U_{l}, k, l \in J_{U}(i, j), i, j\right\} \cap\left(c J c^{*}\right)^{\perp_{q}}=\{0\} .
$$

Given any other Kraus representation of $\Lambda_{q}$, then for every $(i, j)$ there are matrices $\left\{\mu_{r, k}^{i, j}\right\}_{r \in J_{W}(i, j), k \in J_{U}(i, j)}$ such that $\sum_{r \in J_{W}(i, j)} \bar{\mu}_{r, k}^{i, j} \mu_{r, l}^{i, j}=\delta_{k, l}$ for all $k, l \in J_{U}(i, j)$ and $W_{r}=\sum_{k \in J_{U}(i, j)} \mu_{r, k}^{i, j} U_{k}$. It follows that

$$
\operatorname{span}\left\{U_{k}^{*} U_{l}, k, l \in J_{U}(i, j), i, j\right\}=\operatorname{span}\left\{W_{k}^{*} W_{l}, k, l \in J_{W}(i, j), i, j\right\} .
$$

Let us now return to the setting of Lemma1, so that $J=S^{-1}\left(\mathbb{C} \rho_{0}\right)$ with $\rho_{0}=$ $S(\rho)$ for some invertible $\rho \in \mathfrak{S}(\mathcal{A})$ and $S^{*}: \mathcal{A}_{0} \rightarrow \mathcal{A}$ an injective homomorphism. Then $\Phi$ has a minimal decomposition of the form $\Phi=\Lambda_{q} \circ \chi_{d^{1 / 2}}$ and by Lemma 1. there is some $\sigma_{0} \in \mathfrak{S}(\mathcal{A})$, such that $d^{1 / 2}=S^{*}\left(b_{0}\right)$ with $b_{0}$ the unique positive solution of $b_{0} \rho_{0} b_{0}=\sigma_{0}$. Moreover, by the proof of the Lemma,

$$
d^{1 / 2} J d^{1 / 2}=S^{-1}\left(\mathbb{C} \sigma_{0}\right) \subseteq \mathcal{A}_{q},
$$

where $q=\operatorname{supp}(d)=S^{*}\left(\operatorname{supp}\left(b_{0}\right)\right)=S^{*}\left(q_{0}\right)$ with $q_{0}:=\operatorname{supp}\left(\sigma_{0}\right)$.

Corollary 4 In the above setting, $\Phi$ is extremal in $\mathcal{C}_{J}(\mathcal{A}, \mathcal{B})$ if and only if $\Lambda_{q}$ is extremal in $\mathcal{C}_{q J q}\left(\mathcal{A}_{q}, \mathcal{B}\right)$ where $q J q=S^{-1}\left(\mathbb{C} q_{0} \rho_{0} q_{0}\right)$. In particular, if $q=I$ then $\Phi$ is extremal in $\mathcal{C}_{J}(\mathcal{A}, \mathcal{B})$ if and only if $\Lambda$ is.

Proof. By Corollary 3, $\Phi$ is extremal if and only if $\Lambda_{q}$ is an extremal channel and

$$
\operatorname{span}\left\{W_{k}^{*} W_{l}, k, l \in J_{W}(i, j), i, j\right\} \cap S^{*}\left(\left\{\sigma_{0}\right\}^{\perp_{q_{0}}}\right)=\{0\}
$$

for a Kraus representation of $\Lambda_{q}$. Let $\left\{y_{1}, \ldots, y_{m}\right\}$ be a basis of the subspace $\left\{\sigma_{0}\right\}^{\perp_{q_{0}}}$, then $\left\{q_{0}, y_{1}, \ldots, y_{m}\right\}$ is a basis of $\left(\mathcal{A}_{0}\right)_{q_{0}}$. Suppose that (17) holds and let $c_{k, l}$ be some coefficients such that

$$
\sum_{i, j} \sum_{k, l \in J_{W}(i, j)} c_{k, l} W_{k}^{*} W_{l}=S^{*}(x)
$$

for some $x \in\left\{q_{0} \rho_{0} q_{0}\right\}^{\perp_{q_{0}}}$. Let $x=t_{0} q_{0}+\sum_{j} t_{j} y_{j}$. Then since $\Lambda_{q}$ is a channel, we have $\sum_{k} W_{k}^{*} W_{k}=q=S^{*}\left(q_{0}\right)$ and

$$
\sum_{k, l} c_{k, l} W_{k}^{*} W_{l}-t_{0} \sum_{k} W_{k}^{*} W_{k}=S^{*}\left(\sum_{j} b_{j} y_{j}\right) \in S^{*}\left(\left\{\sigma_{0}\right\}^{\perp_{q_{0}}}\right)
$$

Hence $\sum_{k, l} c_{k, l} W_{k}^{*} W_{l}-t_{0} \sum_{k} W_{k}^{*} W_{k}=0$, so that $x=t_{0} q_{0}$. But this is possible only if $x=0$, this implies that

$$
\operatorname{span}\left\{W_{k}^{*} W_{l}, k, l \in J_{W}(i, j), i, j\right\} \cap S^{*}\left(\left\{q_{0} \rho_{0} q_{0}\right\}^{\perp_{q_{0}}}\right)=\{0\}
$$


Similarly, we can prove that (18) implies (17). The statement now follows by Corollary 3 ,

\subsection{Extremality conditions for conjugate maps}

Let $\Phi: \mathcal{A} \rightarrow \mathcal{B}$ be a completely positive map with a minimal Kraus representation $\Phi(a)=\sum_{i, j} \sum_{k \in I(i, j)} V_{k} a V_{k}^{*}$ and let $n_{i, j}=|I(i, j)|, N=\sum_{i, j} n_{i, j}$. Then the conjugate map of $\Phi$ is defined as the map $\Phi^{C}: \mathcal{A} \rightarrow \mathcal{D}:=\bigoplus_{i, j} B\left(\mathbb{C}^{n_{i, j}}\right)$ such that

$$
\Phi^{C}(a)=\bigoplus_{i, j} \sum_{k, l \in I(i, j)} \operatorname{Tr}\left(V_{k} a V_{l}^{*}\right)|k\rangle\langle l|
$$

where $\{|k\rangle\}_{k}$ is a basis of $\mathbb{C}^{N}$ such that $\{|k\rangle, k \in I(i, j)\}$ is a basis of $\mathbb{C}^{n_{i, j}}$. It is clear that $\Phi^{C}$ is completely positive and, since $\operatorname{Tr} \Phi(a)=\operatorname{Tr} \Phi^{C}(a)$ for all $a \in \mathcal{A}, \Phi \in \mathcal{C}_{J}(\mathcal{A}, \mathcal{B})$ if and only if $\Phi^{C} \in \mathcal{C}_{J}(\mathcal{A}, \mathcal{D})$.

Corollary $5 \Phi$ is extremal in $\mathcal{C}_{J}(\mathcal{A}, \mathcal{B})$ if and only if the conjugate map $\Phi^{C}$ satisfies

$$
\Phi^{C}(J)=\mathcal{D}
$$

Proof. Let $\Phi(a)=\sum_{i, j} \sum_{k, l \in I(i, j)} V_{k} a V_{k}^{*}$ be a minimal Kraus representation. Let $D=\oplus_{i, j} \sum_{k, l \in I(i, j)} d_{k, l}\left|e_{k}\right\rangle\left\langle e_{l}\right| \in \mathcal{D}$ and $a \in \mathcal{A}$. Then

$$
\operatorname{Tr} D \Phi^{C}(a)=\operatorname{Tr}\left(\sum_{i, j} \sum_{k, l \in I(i, j)} d_{k, l} V_{k}^{*} V_{l} a\right)
$$

It follows that $\operatorname{Tr} D \Phi^{C}(a)=0$ for all $a \in J$ if and only if $\sum_{i, j} \sum_{k, l \in I(i, j)} d_{k, l} V_{k}^{*} V_{l} \in$ $J^{\perp}$. From this and Corollary 2 , we get that $\Phi$ is extremal if and only if $\Phi^{C}(J)^{\perp}=\{0\}$. Since $\Phi^{C}(J)$ is a subspace in $\mathcal{D}$, this is equivalent with $\Phi^{C}(J)=\mathcal{D}$.

Note that the condition $\Phi(J)=\mathcal{B}$ means that the channel $J \rightarrow \mathcal{B}$ defined by $\Phi$ is surjective.

\subsection{Extremal quantum supermaps}

Since $\mathcal{C}\left(\mathcal{B}_{0}, \ldots, \mathcal{B}_{n}\right)=\frac{1}{c_{n-1}} \mathcal{C}_{J_{n-1}}\left(\mathcal{A}_{n-1}, \mathcal{B}_{n}\right)$, extremality conditions for quantum supermaps can be obtained from the previous sections. For this, we need to describe the subspaces $J_{n-1}^{\perp}$ and their bases. Let $\mathcal{T}(\mathcal{B})$ denote the subspace of traceless elements in $\mathcal{B}$.

Lemma 2 Let $J \subseteq \mathcal{A}$ be a subspace. Then

$$
\left(\operatorname{Tr}_{\mathcal{B}}^{\mathcal{B} \otimes \mathcal{A}}\right)^{-1}(J)=\left(I_{\mathcal{B}} \otimes J\right) \oplus(\mathcal{T}(\mathcal{B}) \otimes \mathcal{A})
$$


Proof. We have $\mathcal{T}(\mathcal{B})=\left\{I_{\mathcal{B}}\right\}^{\perp}$ and $\mathcal{B}=\mathbb{C} I_{\mathcal{B}} \oplus \mathcal{T}(\mathcal{B}), \mathcal{A}=J \oplus J^{\perp}$. Hence

$$
\mathcal{B} \otimes \mathcal{A}=\left(I_{\mathcal{B}} \otimes J^{\perp}\right) \oplus\left(I_{\mathcal{B}} \otimes J\right) \oplus(\mathcal{T}(\mathcal{B}) \otimes \mathcal{A})
$$

Since $L^{\perp}=I_{\mathcal{B}} \otimes J^{\perp}$ by (5), we must have $L=\left(I_{\mathcal{B}} \otimes J\right) \oplus(\mathcal{T}(\mathcal{B}) \otimes \mathcal{A})$.

Let $I_{n}=I_{\mathcal{B}_{n}}, \mathcal{T}_{n}:=\mathcal{T}\left(\mathcal{B}_{n}\right)$ and let $\left\{t_{1}^{n}, \ldots, t_{d_{n}-1}^{n}\right\}$ be some fixed basis of $\mathcal{T}_{n}$, $d_{n}:=\operatorname{dim}\left(\mathcal{B}_{n}\right)$. Moreover, let $c_{n}:=\operatorname{dim}\left(\mathcal{A}_{n}\right)$ and let us fix a basis $\left\{a_{1}^{n}, \ldots, a_{c_{n}}^{n}\right\}$ of $\mathcal{A}_{n}$.

Proposition 3 Put $\mathcal{A}_{0}=\mathcal{B}_{0}$ and $\mathcal{A}_{-1}=\mathbb{C}$. Then

$$
J_{n}^{\perp}=I_{n} \otimes \bigvee_{j=0}^{\left\lfloor\frac{n-1}{2}\right\rfloor}\left[\left(\otimes_{l=1}^{2 j} I_{n-l}\right) \otimes \mathcal{T}_{n-2 j-1} \otimes \mathcal{A}_{n-2 j-2}\right]
$$

Proof. We proceed by induction. For $n=1$, we have already seen in Section 2.1.1 that $J_{1}^{\perp}=I_{\mathcal{B}_{1}} \otimes \mathcal{T}_{0}$. Suppose now that the statement holds for all $m<n$. Note that by (8) and (9), $J_{n}=S_{n}^{-1}\left(I_{\mathcal{B}_{n-1}} \otimes J_{n-2}\right)$, hence by (5) and Lemma 2 ,

$$
J_{n}^{\perp}=I_{\mathcal{B}_{n}} \otimes S_{n-1}^{-1}\left(J_{n-2}^{\perp}\right)=I_{\mathcal{B}_{n}} \otimes\left[\left(I_{\mathcal{B}_{n-1}} \otimes J_{n-2}^{\perp}\right) \oplus\left(\mathcal{T}_{n-1} \otimes \mathcal{A}_{n-2}\right)\right]
$$

It is now easy to finish the proof.

It is clear that the set

$$
\bigcup_{j=0}^{\left\lfloor\frac{n-1}{2}\right\rfloor} \bigcup_{k=1}^{d_{n}-1} \bigcup_{l=1}^{c_{n}}\left\{I_{\mathcal{B}_{n}} \otimes\left(\otimes_{l=1}^{2 j} I_{\mathcal{B}_{n-l}}\right) \otimes t_{k}^{n-2 j-1} \otimes a_{l}^{n-2 j-2}\right\}
$$

is a basis of $J_{n}^{\perp}$.

Remark 1 Let $J \subseteq \mathcal{A}$ and let $\operatorname{Lin}(L)$ be as in (11). We have by Lemma 2 that

$$
\operatorname{Lin}(L)=\left(\operatorname{Tr}_{\mathcal{B}}^{\mathcal{B} \otimes \mathcal{A}}\right)^{-1}\left(\left(J^{T}\right)^{\perp}\right)=\left(I_{\mathcal{B}} \otimes\left(J^{T}\right)^{\perp}\right) \oplus(\mathcal{T}(\mathcal{B}) \otimes \mathcal{A})
$$

so that we can find a basis of $\operatorname{Lin}(L)$ as

$$
\mathbb{B}(\operatorname{Lin}(L)):=\bigcup_{i=1}^{M}\left\{I_{\mathcal{B}} \otimes x_{i}^{T}\right\} \cup \bigcup_{j=1}^{d_{\mathcal{B}}-1} \bigcup_{k=1}^{d_{\mathcal{A}}}\left\{t_{j}^{\mathcal{B}} \otimes a_{k}^{\prime}\right\}
$$

where $\left\{a_{k}^{\prime}\right\}_{k}$ is any basis of $\mathcal{A},\left\{x_{1}, \ldots, x_{M}\right\}$ is a basis of $J^{\perp}$ and $\left\{t_{j}^{\mathcal{B}}\right\}_{j}$ is a basis of $\mathcal{T}(\mathcal{B})$. Theorem 1 can be reformulated as follows: $X$ is extremal if and only if the set

$$
\left\{\left|v_{k}\right\rangle\left\langle v_{l}\right|, k, l \in I(i, j), i, j\right\} \cup \mathbb{B}(\operatorname{Lin}(L))
$$

is linearly independent. Of course, we may chose any other basis of $(\mathcal{B} \otimes \mathcal{A})_{P}$, moreover we may suppose that all the bases consist of self-adjoint elements, since all involved subspaces are self-adjoint. In the case of generalized quantum instruments, we get exactly the extremality condition obtained in [7]. 
Remark 2 Note that an element $X \in \mathcal{C}\left(\mathcal{B}_{0}, \ldots, \mathcal{B}_{n}\right)$ defines many completely positive maps between different input and output spaces. For example a quantum $N$ comb defines a map $B\left(\mathcal{H}_{1} \otimes \cdots \otimes \mathcal{H}_{2 N-2}\right) \rightarrow B\left(\mathcal{H}_{0} \otimes \mathcal{H}_{2 N_{1}}\right)$, but the same element viewed as a quantum supermap defines a map $B\left(\mathcal{H}_{0} \otimes \cdots \otimes \mathcal{H}_{2 N-2}\right) \rightarrow$ $B\left(\mathcal{H}_{2 N-1}\right)$. The Kraus operators, as well as the conjugate map, depend on the choice of this map. The conditions obtained above apply only for the case when the input space is $\mathcal{A}_{n-1}$.

Let now $X \in \mathcal{C}\left(\mathcal{B}_{0}, \mathcal{B}_{1}, \mathcal{B}_{2}\right)$ and let $\Phi_{X}=\Lambda_{q} \circ \chi_{I_{1} \otimes \omega^{1 / 2}}$ be a minimal decomposition of $\Phi_{X}$ for some $\omega \in \mathfrak{S}\left(\mathcal{B}_{0}\right)$. Let $q_{0}:=\operatorname{supp}(\omega)$, so that $q=I_{1} \otimes q_{0}$. Then Corollary 4 implies:

Corollary $6 X$ is extremal in $\mathcal{C}\left(\mathcal{B}_{0}, \mathcal{B}_{1}, \mathcal{B}_{2}\right)$ if and only if $\left(\operatorname{Tr} q_{0}\right)^{-1} X_{\Lambda_{q}}$ is extremal in $\mathcal{C}\left(\left(\mathcal{B}_{0}\right)_{q_{0}}, \mathcal{B}_{1}, \mathcal{B}_{2}\right)$.

In the case of 1-testers, this result was obtained in [7, Theorem 3].

\section{Extremal generalized POVMs}

Let $K \subseteq \mathfrak{S}(\mathcal{A})$ be any convex subset and let $U$ be a finite set, $|U|=m$. A measurement on $K$ with values in $U$ is defined as an affine map from $K$ into the set $P(U)$ of probability measures on $U$. It was proved in 9] that all such measurements can be extended to positive maps $\mathcal{A} \rightarrow \mathbb{C}^{m}$ if and only if $K$ is a section of $\mathfrak{S}(\mathcal{A})$, that is, $K=J \cap \mathfrak{S}(\mathcal{A})$ for $J=\operatorname{span}(K)$.

Any positive map $\Phi: \mathcal{A} \rightarrow \mathbb{C}^{m}$ is given by an $m$-tuple $M=\left(M_{1}, \ldots, M_{m}\right)$ of positive operators, such that $\Phi(a)_{u}=\operatorname{Tr} M_{u} a$ for all $a \in \mathcal{A}$ and if $\Phi$ restricts to a measurement on $K$, we must have $\sum_{u} \operatorname{Tr} M_{u} a=1$ for all $a \in K$, so that

$$
\sum_{u} M_{u} \in\left(I_{\mathcal{A}}+J^{\perp}\right) \cap \mathcal{A}^{+} .
$$

Any $m$-tuple of positive operators with this property is called a generalized POVM (with respect to $J$ ) and the set of all such generalized POVMs will be denoted by $\mathcal{M}_{J}(\mathcal{A}, U)$.

It is quite clear that there is a one-to-one correspondence between $\mathcal{M}_{J}(\mathcal{A}, U)$ and $\mathcal{C}_{J}\left(\mathcal{A}, \mathbb{C}^{m}\right)$, given by

$$
X=\sum_{u \in U}|u\rangle\langle u| \otimes M_{u}^{T}, \quad X \in \mathcal{C}_{J}\left(\mathcal{A}, \mathbb{C}^{m}\right), M \in \mathcal{M}_{J}(\mathcal{A}, U)
$$

Hence we can use the results of the previous section to characterize the extreme points of $\mathcal{M}_{J}(\mathcal{A}, U)$.

Theorem 3 Let $M=\left\{M_{u}, u \in U\right\} \in \mathcal{M}_{J}(\mathcal{A}, U)$ and let $p_{u}=\operatorname{supp}\left(M_{u}\right)$. Then $M$ is extremal in $\mathcal{M}_{J}(\mathcal{A}, U)$ if and only if for any collection $\left\{D_{u}, u \in U\right\}$ with $D_{u} \in \mathcal{A}_{p_{u}}, \sum_{u} D_{u} \in J^{\perp}$ implies $D_{u}=0$ for all $u \in U$. 
Proof. By Theorem 1 .

Corollary 7 Let $p_{u}=\operatorname{supp}\left(M_{u}\right), p=\operatorname{supp}\left(\sum_{i} M_{i}\right)$. If $M$ is extremal in $\mathcal{M}_{J}(\mathcal{A}, U)$, then $\sum_{u} \operatorname{dim}\left(\mathcal{A}_{p_{u}}\right) \leq \operatorname{dim}(p J p)$.

Proof. By Corollary 1.

As a generalized channel, a generalized POVM $M=\left\{M_{u}, u \in U\right\}$ has a minimal decomposition $M=\Lambda_{q} \circ \chi_{c}$, where $c^{*} c=\sum_{u} M_{u}, q=\operatorname{supp}\left(c c^{*}\right)$ and $\Lambda_{q}=\left\{\Lambda_{u}, u \in U\right\}$ is a POVM in $\mathcal{A}_{q}$.

Theorem $4 M$ is extremal in $\mathcal{M}_{J}(\mathcal{A}, U)$ and only if $\Lambda_{q}$ is extremal in $\mathcal{M}_{c J c^{*}}\left(\mathcal{A}_{q}, U\right)$.

Proof. By Theorem 2

\subsection{Extremality of PVM's in $\mathcal{M}_{J}(\mathcal{A}, U)$}

It is well known that any projection-valued measure (PVM) is extremal in the set of POVMs. However, the set of generalized POVMs is larger than the set of POVMs, and an additional condition is needed for extremality of a PVM in $\mathcal{M}_{J}(\mathcal{A}, U)$.

Let $M$ be a PVM. In this case, the range of $M$ generates an abelian subalgebra in $\mathcal{A}$. We denote by $\{M\}^{\prime}$ the commutant of this subalgebra, that is, the set of all $b \in B(\mathcal{H})$, such that $b M_{u}=M_{u} b$ for all $u \in U$.

Proposition 4 Let $M$ be a PVM. Then $M$ is extremal in $\mathcal{M}_{J}(\mathcal{A}, U)$ if and only if

$$
\{M\}^{\prime} \cap J^{\perp}=\{0\}
$$

Proof. If $M$ is a PVM, then $\operatorname{supp}\left(M_{u}\right)=M_{u}$ and for $D \in \mathcal{A}, D \in\{M\}^{\prime}$ if and only if $D=\sum_{u} D_{u}$ with $D_{u}=D M_{u} \in \mathcal{A}_{M_{u}}$. In this case, $D=0$ if and only if $D_{u}=0$ for all $u$. The statement now follows from Theorem 3 ,

Note that combining Proposition 4 with Theorem 4 gives a characterization of extremal generalized POVMs with two outcomes. Indeed, if $M$ is an extremal 2-outcome generalized POVM with minimal decomposition $M=\Lambda_{q} \circ \chi_{c}$, then $\Lambda_{q}$ must be an extremal 2-outcome POVM, hence a PVM.

Corollary 8 Let $U=\{1,2\}$ and let $M \in \mathcal{M}_{J}(\mathcal{A}, U)$ with a minimal decomposition $M=\Lambda_{q} \circ \chi_{c}$. Then $M$ is extremal if and only if $\Lambda_{q}$ is a PVM and

$$
\left\{\Lambda_{q}\right\}^{\prime} \cap\left(c J c^{*}\right)^{\perp_{q}}=\{0\}
$$

Next we look at the condition in Proposition 4 in the setting of Lemma 1. 
Lemma 3 Let $J=S^{-1}\left(\mathbb{C} \rho_{0}\right)$, where $S^{*}$ is an injective homomorphism and $\rho_{0}=S(\rho)$ for some invertible $\rho \in \mathfrak{S}(\mathcal{A})$. Let $M$ be a PVM, then $M$ is extremal in $\mathcal{M}_{J}(\mathcal{A}, U)$ if and only if $S^{*}\left(p_{0}\right) \in\{M\}^{\prime}$ for some projection $p_{0} \in \mathcal{A}_{0}$ implies that $p_{0}=0$ or $I$.

Proof. Let $0 \neq x \in\{M\}^{\prime} \cap J^{\perp}$, then $x=S^{*}(y)$ for some $y \in \mathcal{A}_{0}$ with $\operatorname{Tr} y \rho_{0}=0$ and we may suppose $y=y^{*}$. Let $y=y_{+}-y_{-}$be the decomposition of $y$ into its positive and negative part, then $x_{ \pm}=S^{*}\left(y_{ \pm}\right)$is the decomposition of $x$. Let $p_{+}=\operatorname{supp}\left(y_{+}\right)$, then $S^{*}\left(p_{+}\right)=\operatorname{supp} x_{+} \in\{M\}^{\prime}$. Moreover, since $\rho_{0}$ is invertible, we must have $y_{ \pm} \neq 0$, hence $p_{+} \neq 0, I$.

Conversely, suppose $S^{*}\left(p_{0}\right) \in\{M\}^{\prime}$ for some $p_{0} \neq 0, I$ and let $t=\operatorname{Tr} \rho_{0} p_{0}$. Put $y:=p_{0}-\frac{t}{1-t}\left(I-p_{0}\right)$, then $y \neq 0$ and $S^{*}(y) \in\{M\}^{\prime} \cap J^{\perp}$.

Example 2 Let $K=\operatorname{Diag}_{\lambda}$ as in Example 1 and let $M \in \mathcal{M}_{J_{\lambda}}\left(M_{n}(\mathbb{C}), U\right)$. Then $M$ has a minimal decomposition of the form $M=\Lambda_{q} \circ \chi_{d^{1 / 2}}$ with $d=$ $\sum_{i} d_{i}|i\rangle\langle i|, d_{i}=\mu_{i} / \lambda_{i}$ for some probability vector $\mu$. Let $I=\left\{i, d_{i}>0\right\}$, then $q=p_{I}:=\sum_{i \in I}|i\rangle\langle i|$. Let us denote by $M_{I}(\mathbb{C})$ the subalgebra in $M_{n}(\mathbb{C})$ generated by the matrix units $\{|i\rangle\langle j|, i, j \in I\}$, then $\Lambda_{q}$ is a POVM on $M_{I}(\mathbb{C})$. By Corollary $4, M$ is extremal if and only if $\Lambda_{q}$ is extremal in $\mathcal{M}_{p_{I} J p_{I}}\left(M_{I}(\mathbb{C}), U\right)$.

Suppose that $\Lambda_{q}$ is a PVM. Then $M$ is extremal if and only if there is no nonempty $J \subsetneq I$ such that $p_{J} \in\left\{\Lambda_{q}\right\}^{\prime}$.

\subsection{Extremal 1-testers}

An $n$-tester on $\left(\mathcal{H}_{0}, \ldots, \mathcal{H}_{2 n-1}\right)$ is a map that defines a measurement on the set of $n$-combs on some finite dimensional Hilbert spaces $\mathcal{H}_{0}, \ldots, \mathcal{H}_{2 n-1}$, hence it is a (constant multiple of) a generalized POVM with respect to $J_{2 n-1}\left(\mathcal{H}_{0}, \ldots, \mathcal{H}_{2 n-1}\right)$. As generalized channels, $n$-testers are elements of $\mathcal{C}\left(\mathcal{H}_{0}, \ldots, \mathcal{H}_{2 n-1}, \mathbb{C}^{m}\right)$.

In particular, 1-testers define measurements on the set of channels $\mathcal{C}\left(\mathcal{H}_{0}, \mathcal{H}_{1}\right)$. The 1-testers were introduced in [2], note that these appeared independently in [11, under the name PPOVMs (process POVMs). Hence a 1-tester is a collection of operators $M=\left\{M_{u}, u \in U\right\}, M_{u} \in B\left(\mathcal{H}_{1} \otimes \mathcal{H}_{0}\right)^{+}$, with $\sum_{u} M_{u}=$ $I_{\mathcal{H}_{1}} \otimes \omega$ for some $\omega \in \mathfrak{S}\left(\mathcal{H}_{0}\right)$. It follows that $M$ has a minimal decomposition of the form $M=\Lambda \circ \chi_{I_{1} \otimes \omega^{1 / 2}}$ and $\Lambda$ is a POVM on $B\left(\mathcal{H}_{1} \otimes q_{0} \mathcal{H}_{0}\right)$, with $q_{0}=\operatorname{supp}(\omega)$. Moreover, by Corollary 6, $M$ is extremal if and only if $\operatorname{Tr} q_{0}^{-1} \Lambda$ is an extremal 1-tester on $\left(q_{0} \mathcal{H}_{0}, \mathcal{H}_{1}\right)$.

Let us summarize the results of the previous section for 1-testers.

Proposition 5 (i) Let $p_{u}=\operatorname{supp}\left(M_{u}\right)$. Then $M$ is an extremal 1-tester on $\left(\mathcal{H}_{0}, \mathcal{H}_{1}\right)$ if and only if $D_{u} \in B\left(p_{u}\left(\mathcal{H}_{1} \otimes \mathcal{H}_{0}\right)\right), \sum_{u} D_{u}=I_{\mathcal{H}_{1}} \otimes x$ with $\operatorname{Tr} x=0$ implies $D_{u}=0$ for all $u$.

(ii) Suppose that $\Lambda$ is a PVM. Then $M$ is extremal if and only if $I_{\mathcal{H}_{1}} \otimes p \in\{\Lambda\}^{\prime}$ for some projection $p$ on $q_{0} \mathcal{H}_{0}$ implies $p=0$ or $p=q_{0}$. 
(iii) Let $U=\{1,2\}$, then $M$ ix extremal if and only if $\Lambda=\left(\Lambda_{1}, \Lambda_{2}\right)$ is a PVM and $\Lambda_{1}$ commutes with no projection of the form $I_{\mathcal{H}_{1}} \otimes p$ with $p \neq 0, q_{0}$.

Example 3 ( Extremal 2-outcome qubit 1-testers) Extremal 1-testers with 2 outcomes for $\operatorname{dim}\left(\mathcal{H}_{1}\right)=\operatorname{dim}\left(\mathcal{H}_{0}\right)=2$ were described in 7 . We show that using our results, this is very easily achieved and extended to the case $\operatorname{dim}\left(\mathcal{H}_{1}\right)=n \geq 2$.

So let $M=\left(M_{1}, M_{2}\right)$ be a 1-tester on $\left(\mathcal{H}_{0}, \mathcal{H}_{1}\right)$ with $\operatorname{dim}\left(\mathcal{H}_{0}\right)=2$ and let $M_{1}+M_{2}=I_{1} \otimes \omega$. Suppose first that $\operatorname{rank}(\omega)=1$, then $\omega=|\varphi\rangle\langle\varphi|$ for some $\varphi \in \mathcal{H}_{0}$ and then $M_{u}=\Lambda_{u}=N_{u} \otimes|\varphi\rangle\langle\varphi|$ for some POVM $N$ on $B\left(\mathcal{H}_{1}\right)$. Since any subspace in a one-dimensional space is trivial, $M$ is extremal if and only if $\Lambda$ (and hence also $M$ ) is a PVM.

Suppose $\operatorname{rank}(\omega)=2$, then $q_{0}=I_{0}$. Any nontrivial projection $p$ on $\mathcal{H}_{0}$ is rank one, $p=\left|\psi_{0}\right\rangle\left\langle\psi_{0}\right|$. Hence $M$ is extremal if and only if $\Lambda$ is a PVM, such that $\Lambda_{1}$ is not of the form

$$
\Lambda_{1}=e \otimes\left|\psi_{0}\right\rangle\left\langle\psi_{0}|+f \otimes| \psi_{0}^{\perp}\right\rangle\left\langle\psi_{0}^{\perp}\right|
$$

where $\psi_{0}, \psi_{0}^{\perp} \in \mathcal{H}_{0}$ are unit vectors such that $\left\langle\psi_{0}, \psi_{0}^{\perp}\right\rangle=0$ and $e, f$ are projections in $B\left(\mathcal{H}_{1}\right)$.

\section{Concluding remarks}

We have obtained a set of extremality conditions for generalized channels. These conditions are not easily checked, in fact, in the case of quantum supermaps the conditions are quite complicated. However, for the case of a generalized POVM consisting of a PVM combined with a simple generalized channel, we found a simpler condition.

As already mentioned in the Introduction, there is no one-to-one correspondence between generalized channels and channels defined on the subspace $J$. In particular, measurements on a section of the state space correspond to equivalence classes of generalized POVMs, because in general, there are many generalized POVMs giving the same probabilities for all elements in the section. Therefore, extremal generalized POVMs do not necessarily correspond to extremal measurements on sections. The question of extremal measurements will be addressed in a forthcoming paper.

\section{References}

[1] G. Chiribella, G. M. D'Ariano, P. Perinotti, Quantum circuit architecture, Phys. Rev. Lett. 101 (2008), 060401

[2] G. Chiribella, G. M. D'Ariano, and P. Perinotti, Memory effects in quantum channel discrimination, Phys. Rev. Lett. 101, 180501 (2008). 
[3] G. Chiribella, G. M. D'Ariano, P. Perinotti, Transforming quantum operations: quantum supermaps, Europhysics Letters 83 (2008), 30004

[4] G. Chiribella, G. M. D'Ariano, P. Perinotti, Theoretical framework for quantum networks, Phys. Rev. A 80 (2009), 022339

[5] G. Chiribella, G. M. D'Ariano, P. Perinotti, D. M. Schlingemann, R. F. Werner, A short impossibility proof of Quantum Bit Commitment, arXiv:0905.3801

[6] M.D. Choi, Completely positive maps on complex matrices, Lin. Alg. Appl. 10 (1975), 285-290

[7] G. M. D'Ariano, P. Perinotti, M. Sedlak, Extremal quantum protocols, J. Math. Phys. 52, 082202 (2011), arXiv:1101.4889

[8] G. Gutoski, J. Watrous, Toward a general theory of quantum games, . Proceedings of the 39th ACM Symposium on Theory of Computing, pages 565574,2007

[9] A. Jenčová, Generalized channels: channels for convex subsets of the state space, J. Math. Phys. 53, 012201 (2011), arXiv:1105.1899

[10] T. Rockafellar, Convex Analysis, Princeton University Press, 1970 Princeton

[11] M. Ziman, Process POVM: A mathematical framework for the description of process tomography experiments, Phys. Rev. A 77, 062112 (2008) 